\title{
An efficient synthesis of 8-aryl-9H-cyclopenta[a][4,7]phenanthroline derivatives catalyzed by iodine
}

\author{
Ming-Yue Yin, ${ }^{a}$ Mei-Mei Zhang, ${ }^{b}$ Wei Wang, ${ }^{a}$ Yu-Ling Li, ${ }^{a}$ and Xiang-Shan Wang $* a$ \\ ${ }^{a}$ School of Chemistry and Chemical Engineering, Jiangsu Key Laboratory \\ of Green Synthetic Chemistry for Functional Materials, Xuzhou Normal University, \\ Xuzhou, Jiangsu 221 116, P.R. China \\ ${ }^{b}$ The Key Laboratory of Biotechnology on Medical Plant of Jiangsu Province, \\ Xuzhou Normal University, Xuzhou, Jiangsu 221 116, P.R. China \\ E-mail:xxwang1974@yahoo.com
}

\begin{abstract}
A series of 10,11-dihydro-8-aryl-9H-cyclopenta[a][4,7]phenanthroline derivatives was prepared by a three-component reaction of aromatic aldehyde, quinolin-6-amine and cyclopentanone using iodine as catalyst. The structure of $\mathbf{4 e}$ is confirmed by X-ray diffraction analysis, and the crystal structure is discussed in detail. Compared to the previous method, this iodine-catalyzed procedure has the advantages of mild reaction conditions, good yields and operational simplicity.
\end{abstract}

Keywords: Phenanthroline, quinolin-6-amine, cyclopentanone, iodine, synthesis

\section{Introduction}

In recent years, multi-component reactions (MCRs) have become important tools in modern preparative synthetic chemistry because they increase the efficiency by combining several operational steps without isolation of intermediates or changing the reaction conditions. ${ }^{1}$ MCRs have emerged as valuable tools for the preparation of structurally diverse chemical libraries of drug-like heterocyclic compounds. ${ }^{2}$ Owing to their convergence and productivity, the MCRs have attracted considerable attention from the organic synthetic chemistry point of view. ${ }^{3}$

The phenanthrolines and their derivatives are well known compounds for their metallic complexes. The latter possess remarkable physiological and pharmacological activities. Their activities include anticancer (copper(II)), ${ }^{4}$ antiinflammatory (copper(II)), ${ }^{5}$ antitumor (Pt(II)), ${ }^{6}$ antimicrobial (copper(II)), ${ }^{7}$ and antibacterial activity (Y(III)). ${ }^{8}$ In addition, it was reported that phenanthroline derivatives themselves also had commendable antitumor activity. ${ }^{9}$

Kozlov et al. ${ }^{10}$ reported that Schiff base containing quinoline fragment, could react with cyclopentanone or cyclohexanone to produce 4,7-phenanthrolines, which was promoted by $\mathrm{HCl}$, 
in 2003. However, this method suffered from the drawbacks of low yields (27-41\%) and multistep reactions.

In view of the importance of phenanthroline derivatives and as a continuation of our research devoted to the development of new methods for the preparation of heterocycles via multi-component reactions catalyzed by iodine, ${ }^{10}$ herein we describe the synthesis of 8 -aryl-9Hcyclopenta $[a][4,7]$ phenanthroline derivatives by a reaction of aromatic aldehyde, and quinolin-6amine and cyclopentanone in THF catalyzed by iodine.

\section{Results and Discussion}

Treatment of aromatic aldehyde 1, quinolin-6-amine $\mathbf{2}$ and cyclopentanone $\mathbf{3}$ in THF in the presence of $5 \mathrm{~mol} \%$ iodine at reflux condition afforded the corresponding 10,11-dihydro-8-aryl$9 H$-cyclopenta[a][4,7]phenanthroline 4 in good yields (Scheme 1).

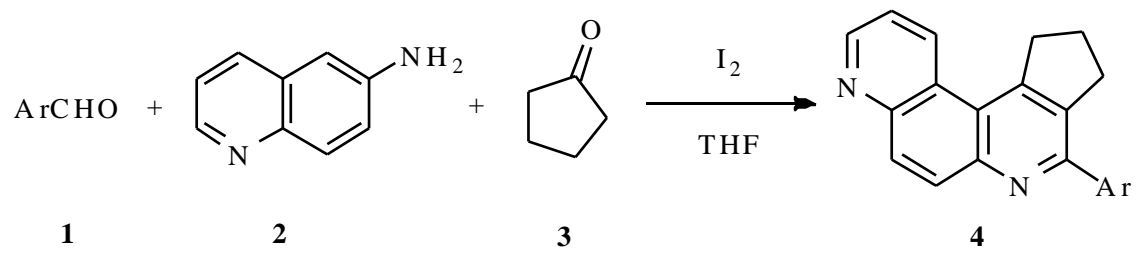

Scheme 1. The reaction of $\mathbf{1 , 2}$ and cyclopentanone 3 .

Table 1. Synthesis of $\mathbf{4 a}$ at different reaction conditions ${ }^{a}$

\begin{tabular}{lllll}
\hline Entry & $\mathrm{T} /{ }^{\circ} \mathrm{C}$ & solvent & $\mathrm{I}_{2} / \mathrm{mol} \%$ & Yield $^{b} \%$ \\
\hline 1 & reflux & $\mathrm{THF}$ & 0 & 0 \\
2 & r.t. & $\mathrm{THF}$ & 5 & trace \\
3 & 50 & $\mathrm{THF}$ & 5 & 72 \\
4 & reflux & $\mathrm{THF}$ & 5 & 82 \\
5 & reflux & $\mathrm{THF}$ & 1 & 68 \\
6 & reflux & $\mathrm{THF}$ & 10 & 82 \\
7 & reflux & $\mathrm{CHCl}$ & 5 & 74 \\
8 & reflux & benzene & 5 & 80 \\
9 & reflux & $\mathrm{CH}_{3} \mathrm{CN}$ & 5 & 78 \\
10 & 80 & $\mathrm{DMF}$ & 5 & 65 \\
\hline
\end{tabular}

${ }^{a}$ Reaction conditions: solvent $(10 \mathrm{~mL})$, 4-bromobenzaldehyde (0.370 g, $\left.2 \mathrm{mmol}\right), 2 \mathrm{mmol}$ quinolin-6-amine (0.288 g, $2 \mathrm{mmol})$, cyclopentanone (0.176 g, $2.1 \mathrm{mmol}),{ }^{b}$ Isolated yields. 
Using the conversion of 2-bromobenzaldehyde 1a, quinolin-6-amine and cyclopentanone as a model, several parameters were explored as shown in Table 1. the reaction did not take place at reflux in the absence of iodine (Table 1, Entry 1). Similar reactions were attempted in the presence of 1,5 and $10 \mathrm{~mol} \%$ of $\mathrm{I}_{2}$. The results from Table 1 show that $5 \mathrm{~mol} \% \mathrm{I}_{2}$ at reflux in THF is sufficient to initiate the reaction. Higher loading of the catalyst had no significant influence on the reaction yield. (Table 1, entries 4-6). The yield of $\mathbf{4 a}$ was also dependent on temperature (entries 2-4), proceeding smoothly at reflux. Different solvents were also tested, and THF appeared to be the best medium for this transformation (entry $4 \mathrm{vs}$. 7-10).

This process can tolerate both electron-donating (alkyl and alkoxy-) and electronwithdrawing (halogen) substituents on the aromatic aldehydes (Table 1). In all cases, the reactions proceeded efficiently at reflux to afford the corresponding cyclopenta[a][4,7]phenanthrolines in good yields. The structures of the products $\mathbf{4 a - 4 j}$ were characterized by IR, ${ }^{1} \mathrm{H}$ NMR and HRMS, all the data were good agreement to their structures. The structure of $\mathbf{4 e}$ was additionally confirmed by X-ray diffraction analysis. Crystal data for $4 \mathbf{e}: \mathrm{C}_{21} \mathrm{H}_{15} \mathrm{FN}_{2} ; \mathrm{M}=$ 314.35 , Orange block crystals, $0.655 \times 0.410 \times 0.087 \mathrm{~mm}$, Monoclinic, space group P 21/c, $a=$ 8.6497 (2), $b=10.5419$ (2), $c=17.2544$ (3) $\AA, \beta=102.823$ (1) ${ }^{\circ}, V=1534.09(5)^{3}, Z=4, D_{\mathrm{c}}=$ $1.361 \mathrm{~g} \cdot \mathrm{cm}^{-3} \cdot F(000)=656, \mu(\mathrm{MoK} \alpha)=0.089 \mathrm{~mm}^{-1}$. Intensity data were collected on Rigaku Mercury diffractometer with graphite monochromated $\operatorname{Mo} K \alpha$ radiation $(\lambda=0.71070 \AA)$ using $\omega$ scan mode with $2.28^{\circ}<\theta<25.20^{\circ}$. 2767 unique reflections were measured and 2180 reflections with $I>2 \sigma(I)$ were used in the refinement. Structure solved by direct methods and expanded using Fourier techniques. The final cycle of full-matrix least squares technique to $R=$ 0.0349 and $w R=0.0467$. The crystal structure of $\mathbf{4 e}$ is shown in the Figure 1 , and the selected bond lengths and angles are listed in Table 3 and Table 4, respectively.

Table 2. Synthetic results of $\mathbf{4}$ catalyzed by iodine in $\mathrm{THF}^{a}$

\begin{tabular}{lllll}
\hline Entry & $\mathrm{Ar}$ & Products & Time (h) & Isolated Yields (\%) \\
\hline 1 & $4-\mathrm{BrC}_{6} \mathrm{H}_{4}$ & $\mathbf{4 a}$ & 22 & 82 \\
2 & $3-\mathrm{ClC}_{6} \mathrm{H}_{4}$ & $\mathbf{4 b}$ & 26 & 73 \\
3 & $4-\mathrm{ClC}_{6} \mathrm{H}_{4}$ & $\mathbf{4 c}$ & 26 & 81 \\
4 & $3-\mathrm{FC}_{6} \mathrm{H}_{4}$ & $\mathbf{4 d}$ & 18 & 67 \\
5 & $4-\mathrm{FC}_{6} \mathrm{H}_{4}$ & $\mathbf{4 e}$ & 20 & 78 \\
6 & $4-\mathrm{CH}_{3} \mathrm{C}_{6} \mathrm{H}_{4}$ & $\mathbf{4 f}$ & 16 & 72 \\
7 & $4-\mathrm{CH}_{3} \mathrm{OC}_{6} \mathrm{H}_{4}$ & $\mathbf{4 g}$ & 23 & 79 \\
8 & $2,3-\left(\mathrm{CH}_{3} \mathrm{O}\right)_{2} \mathrm{C}_{6} \mathrm{H}_{3}$ & $\mathbf{4 h}$ & 26 & 68 \\
9 & $3,4-\left(\mathrm{CH}_{3}\right)_{2} \mathrm{C}_{6} \mathrm{H}_{3}$ & $\mathbf{4 i}$ & 28 & 73 \\
10 & $3,4-\mathrm{OCH}_{2} \mathrm{OC}_{6} \mathrm{H}_{3}$ & $\mathbf{4 j}$ & 22 & 75 \\
\hline
\end{tabular}

${ }^{\text {a }}$ Reagents and conditions: 1 (2.0 mmol), quinolin-6-amine $(0.288 \mathrm{~g}, 2.0 \mathrm{mmol})$, cyclopentanone (0.176 g, $2.1 \mathrm{mmol}), \mathrm{I}_{2}(0.1 \mathrm{mmol}, 0.026 \mathrm{~g})$, THF (10 mL). 


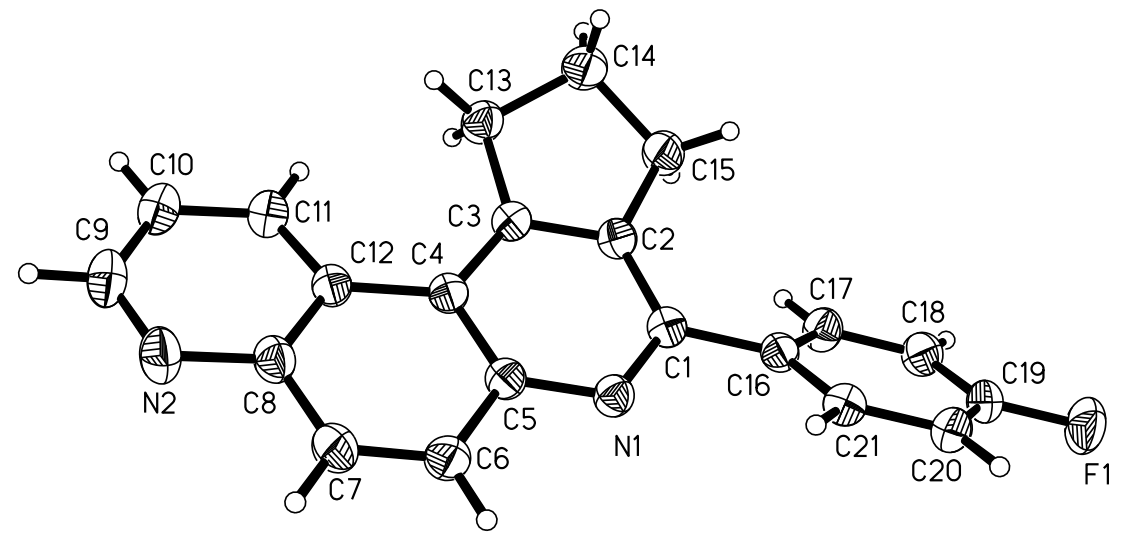

Figure 1. The crystal structure of $\mathbf{4 e}$.

Table 3. The selected bond lengths $(\AA)$ of $\mathbf{4 e}$

\begin{tabular}{ll|ll|ll}
\hline Bond & Length $(\AA)$ & Bond & Length $(\AA)$ & Bond & Length $(\AA)$ \\
\hline $\mathrm{N}(1)-\mathrm{C}(1)$ & $1.3329(16)$ & $\mathrm{C}(2)-\mathrm{C}(15)$ & $1.5118(19)$ & $\mathrm{C}(8)-\mathrm{N}(2)$ & $1.3565(17)$ \\
$\mathrm{N}(1)-\mathrm{C}(5)$ & $1.3551(16)$ & $\mathrm{C}(12)-\mathrm{C}(11)$ & $1.4016(19)$ & $\mathrm{C}(8)-\mathrm{C}(7)$ & $1.424(2)$ \\
$\mathrm{C}(1)-\mathrm{C}(2)$ & $1.4051(18)$ & $\mathrm{C}(12)-\mathrm{C}(8)$ & $1.414(2)$ & $\mathrm{N}(2)-\mathrm{C}(9)$ & $1.317(2)$ \\
$\mathrm{C}(1)-\mathrm{C}(16)$ & $1.4847(17)$ & $\mathrm{C}(3)-\mathrm{C}(13)$ & $1.5088(18)$ & $\mathrm{C}(10)-\mathrm{C}(11)$ & $1.3634(19)$ \\
$\mathrm{C}(4)-\mathrm{C}(3)$ & $1.4132(18)$ & $\mathrm{C}(5)-\mathrm{C}(6)$ & $1.4320(19)$ & $\mathrm{C}(10)-\mathrm{C}(9)$ & $1.375(2)$ \\
$\mathrm{C}(4)-\mathrm{C}(5)$ & $1.4132(18)$ & $\mathrm{F}(1)-\mathrm{C}(19)$ & $1.3655(15)$ & $\mathrm{C}(13)-\mathrm{C}(14)$ & $1.527(2)$ \\
$\mathrm{C}(4)-\mathrm{C}(12)$ & $1.4540(18)$ & $\mathrm{C}(6)-\mathrm{C}(7)$ & $1.3425(19)$ & $\mathrm{C}(14)-\mathrm{C}(15)$ & $1.524(2)$ \\
$\mathrm{C}(2)-\mathrm{C}(3)$ & $1.3789(18)$ & & & & \\
\hline
\end{tabular}

Table 4. The selected bond angles of $4 \mathbf{e}$

\begin{tabular}{lc||lc||lc}
\hline \multicolumn{1}{c|}{ Angle } & \multicolumn{1}{c|}{$\left({ }^{\circ}\right)$} & \multicolumn{1}{|c||}{ Angle } & \multicolumn{1}{c}{$\left(^{\circ}\right)$} & \multicolumn{1}{|c}{ Angle } & $\left(^{\circ}\right)$ \\
\hline $\mathrm{C}(1)-\mathrm{N}(1)-\mathrm{C}(5)$ & $118.94(11)$ & $\mathrm{C}(11)-\mathrm{C}(12)-\mathrm{C}(8)$ & $115.97(12)$ & $\mathrm{C}(12)-\mathrm{C}(8)-\mathrm{C}(7)$ & $119.69(12)$ \\
$\mathrm{N}(1)-\mathrm{C}(1)-\mathrm{C}(2)$ & $120.89(11)$ & $\mathrm{C}(11)-\mathrm{C}(12)-\mathrm{C}(4)$ & $124.99(13)$ & $\mathrm{C}(9)-\mathrm{N}(2)-\mathrm{C}(8)$ & $117.56(13)$ \\
$\mathrm{N}(1)-\mathrm{C}(1)-\mathrm{C}(16)$ & $115.96(11)$ & $\mathrm{C}(8)-\mathrm{C}(12)-\mathrm{C}(4)$ & $119.03(12)$ & $\mathrm{C}(6)-\mathrm{C}(7)-\mathrm{C}(8)$ & $121.41(13)$ \\
$\mathrm{C}(2)-\mathrm{C}(1)-\mathrm{C}(16)$ & $123.12(11)$ & $\mathrm{C}(2)-\mathrm{C}(3)-\mathrm{C}(4)$ & $120.01(12)$ & $\mathrm{C}(18)-\mathrm{C}(19)-\mathrm{F}(1)$ & $118.62(13)$ \\
$\mathrm{C}(21)-\mathrm{C}(16)-\mathrm{C}(1)$ & $119.86(11)$ & $\mathrm{C}(2)-\mathrm{C}(3)-\mathrm{C}(13)$ & $109.85(11)$ & $\mathrm{F}(1)-\mathrm{C}(19)-\mathrm{C}(20)$ & $118.33(13)$ \\
$\mathrm{C}(17)-\mathrm{C}(16)-\mathrm{C}(1)$ & $122.23(12)$ & $\mathrm{C}(4)-\mathrm{C}(3)-\mathrm{C}(13)$ & $130.08(11)$ & $\mathrm{C}(11)-\mathrm{C}(10)-\mathrm{C}(9)$ & $119.04(15)$ \\
$\mathrm{C}(3)-\mathrm{C}(4)-\mathrm{C}(5)$ & $115.36(11)$ & $\mathrm{N}(1)-\mathrm{C}(5)-\mathrm{C}(4)$ & $124.18(12)$ & $\mathrm{C}(10)-\mathrm{C}(11)-\mathrm{C}(12)$ & $120.31(14)$ \\
$\mathrm{C}(3)-\mathrm{C}(4)-\mathrm{C}(12)$ & $125.68(12)$ & $\mathrm{N}(1)-\mathrm{C}(5)-\mathrm{C}(6)$ & $116.14(12)$ & $\mathrm{N}(2)-\mathrm{C}(9)-\mathrm{C}(10)$ & $123.87(14)$ \\
$\mathrm{C}(5)-\mathrm{C}(4)-\mathrm{C}(12)$ & $118.86(11)$ & $\mathrm{C}(4)-\mathrm{C}(5)-\mathrm{C}(6)$ & $119.69(12)$ & $\mathrm{C}(3)-\mathrm{C}(13)-\mathrm{C}(14)$ & $103.26(11)$ \\
$\mathrm{C}(3)-\mathrm{C}(2)-\mathrm{C}(1)$ & $120.28(12)$ & $\mathrm{C}(7)-\mathrm{C}(6)-\mathrm{C}(5)$ & $121.11(13)$ & $\mathrm{C}(15)-\mathrm{C}(14)-\mathrm{C}(13)$ & $105.01(12)$ \\
$\mathrm{C}(3)-\mathrm{C}(2)-\mathrm{C}(15)$ & $110.49(12)$ & $\mathrm{N}(2)-\mathrm{C}(8)-\mathrm{C}(12)$ & $123.10(14)$ & $\mathrm{C}(2)-\mathrm{C}(15)-\mathrm{C}(14)$ & $102.85(11)$ \\
$\mathrm{C}(1)-\mathrm{C}(2)-\mathrm{C}(15)$ & $129.14(12)$ & $\mathrm{N}(2)-\mathrm{C}(8)-\mathrm{C}(7)$ & $117.21(13)$ & & \\
\hline
\end{tabular}


The X-ray diffraction analysis of $\mathbf{4 e}$ indicates that the five-numbered ring $(\mathrm{C} 2, \mathrm{C} 3$, and $\mathrm{C} 13 \sim \mathrm{C} 15)$ is slightly distorted, forming an envelope conformation: the atoms C13, C2, C15 and C13 are coplanar, while the atom C14 deviates from the defined plane by $0.451(3) \AA$. The pyridine ring nearly parallel to the above basal plane and adjacent quinoline ring, forming the dihedral angles of $5.2(1)$ and $6.0(1)^{\circ}$, respectively, and make a dihedral angle of $33.6(1)^{\circ}$ to the benzene ring (C16 C21).

The X-ray diffraction analysis of $\mathbf{4 e}$ reveals that there is no hydrogen bond in the crystal structure. It should be noted that there is intermolecular $\pi$ - $\pi$ interaction between the two neighboring benzene rings ( $\mathrm{C} 4-\mathrm{C} 8$ and $\mathrm{C} 12$ ), symmetry code: $-x, 1-y,-z)$, which are parallel to each other. The centroid-to-centroid distance, plane-plane distance and displacement distance are 3.996(3), 3.507 and $1.917 \AA$, respectively, which indicate the existence of intermolecular $\pi-\pi$ interaction. The above $\pi$ - $\pi$ interactions link the adjacent molecules forming dimers along $b$ axis (Figure 2).

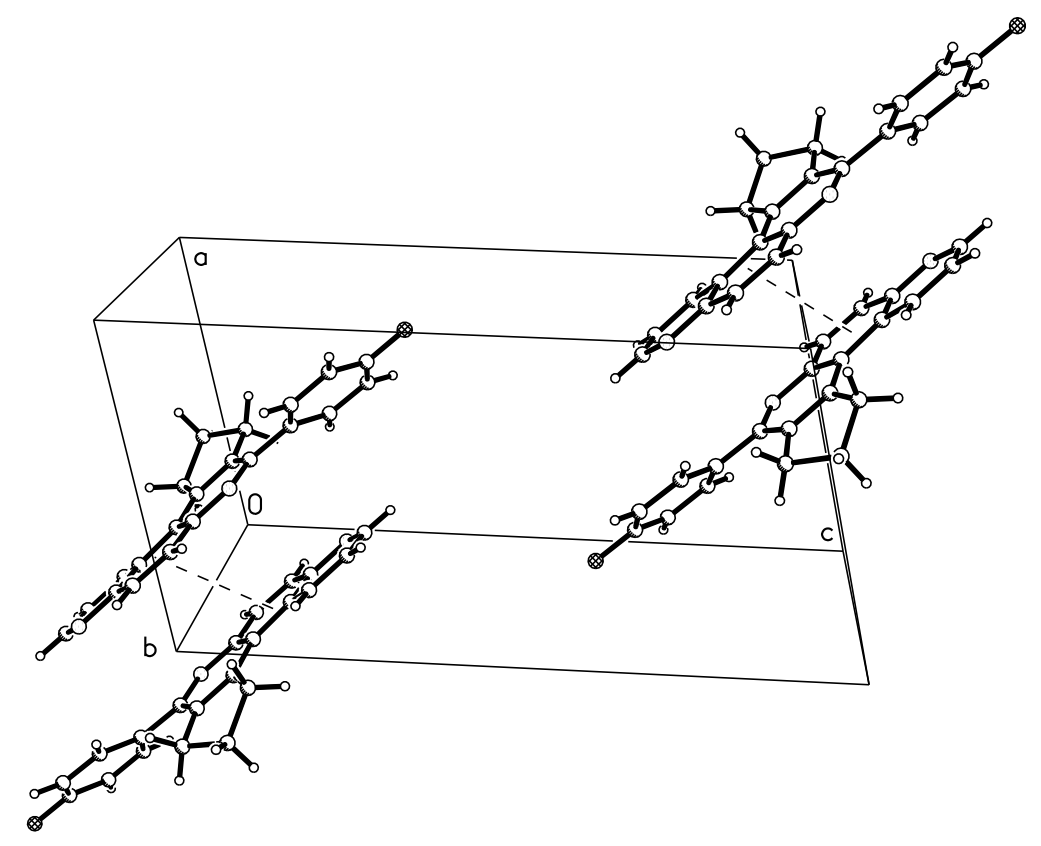

Figure 2. The $\pi-\pi$ interaction in the crystal structure

According to the literature, ${ }^{12}$ we think that iodine catalyzes the reaction as a mild Lewis acid. The mechanism was tentatively proposed as shown in Scheme 2. The Schiff base I may be formed by the reaction of aromatic aldehyde and quinolin-6-amine firstly. And then iminoDiels-Alder reaction between the iodine-activated Schiff base II and enol form of $\mathbf{3}$ takes place to form intermediate III, followed by isomerization and dehydration results in dihydroquinoline IV, which is further oxidized by air to afford aromatized cyclopenta $[a][4,7]$ phenanthroline 4 . 


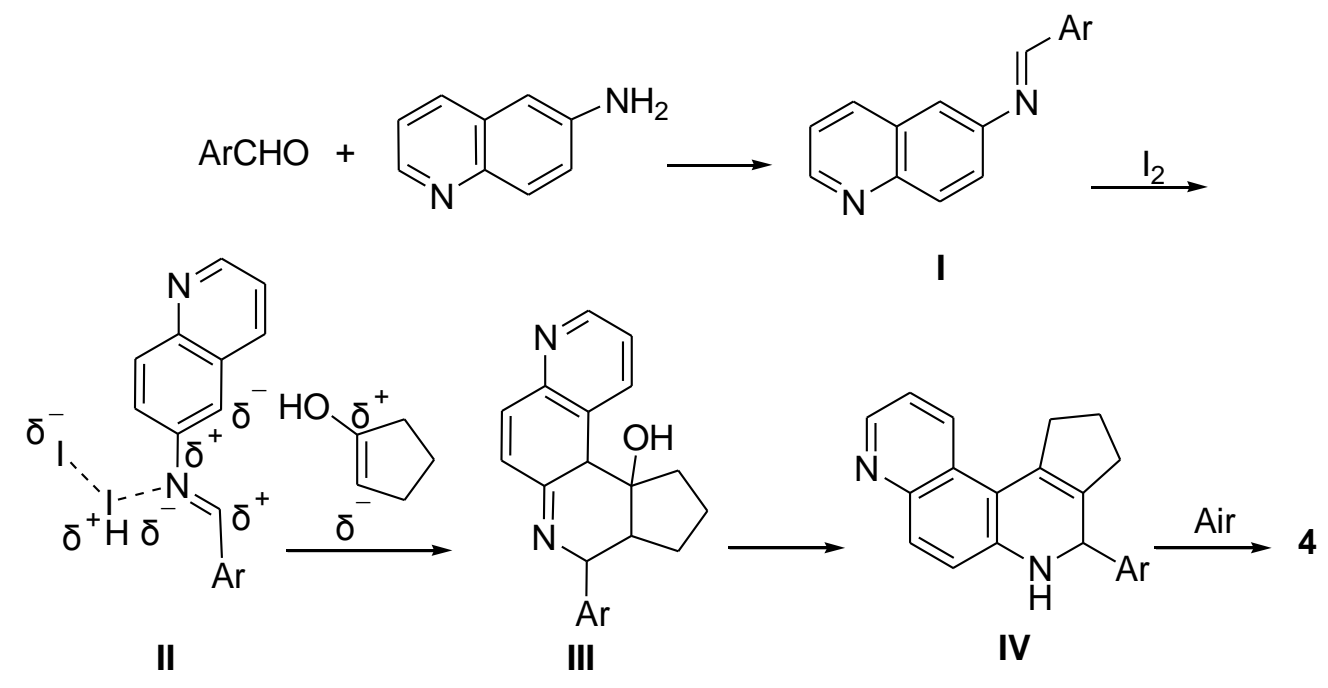

Scheme 2. The possible reaction mechanism of the products 4 .

\section{Conclusions}

In conclusion, we found a mild and efficient method for the synthesis of 8-aryl-9Hcyclopenta $[a][4,7]$ phenanthroline derivatives via three-component reaction of aromatic aldehyde, quinolin-6-amine and cyclopentanone using iodine as catalyst. The features of this procedure are mild reaction conditions, good yields and operational simplicity.

\section{Experimental Section}

General. Melting points were determined in open capillaries and are uncorrected. IR spectra were recorded on a Tensor 27 spectrometer in $\mathrm{KBr}$ pellet. ${ }^{1} \mathrm{H}$ NMR spectra was obtained from a solution in $\mathrm{CDCl}_{3}$ with $\mathrm{Me}_{4} \mathrm{Si}$ as internal standard using a Bruker-400 spectrometer. HRMS analyses were carried out using a Bruker-micro-TOF-Q-MS analyzer.

Procedure for the synthesis of cyclopenta[a][4,7]phenanthrolines (4). A dry $50 \mathrm{~mL}$ flask was charged with aromatic aldehyde $(2.0 \mathrm{mmol})$, quinolin-6-amine $(0.288 \mathrm{~g}, 2.0 \mathrm{mmol})$, cyclopentanone $(0.176 \mathrm{~g}, 2.1 \mathrm{mmol})$, THF $(10 \mathrm{~mL})$ and $\mathrm{I}_{2}(0.1 \mathrm{mmol}, 0.026 \mathrm{~g})$. The reaction mixture was stirred at reflux for 16-28 h, and then a small amount of DMF was added to the mixture, until all the precipitate was dissolved. The products $\mathbf{4}$ were obtained by filtration, when the mixture was allowed to cool down to room temperature.

8-(4-Bromophenyl)-10,11-dihydro-9H-cyclopenta[a][4,7]phenanthroline (4a). Mp 211-212 ${ }^{\circ} \mathrm{C} ;{ }^{1} \mathrm{H}$ NMR $\left(\mathrm{CDCl}_{3}, 400 \mathrm{MHz}\right): \delta_{\mathrm{H}} 2.96 \sim 2.37\left(\mathrm{~m}, 2 \mathrm{H}, \mathrm{CH}_{2}\right), 3.28\left(\mathrm{t}, J 7.6 \mathrm{~Hz}, 2 \mathrm{H}, \mathrm{CH}_{2}\right), 3.77$ (t, J 7.2 Hz, 2H, CH $), 7.60$ (dd, J 8.4 Hz, J' $4.4 \mathrm{~Hz}, 1 \mathrm{H}, \mathrm{ArH}), 7.66$ (d, J 8.8 Hz, 2H, ArH), 7.80 (d, $J 8.0 \mathrm{~Hz}, 2 \mathrm{H}, \mathrm{ArH}), 8.20$ (d, J 9.6 Hz, 1H, ArH), 8.32 (d, J 9.6 Hz, 1H, ArH), 8.99 9.02 (m, 
2H, ArH). IR (KBr): $v$ 3030, 2953, 2932, 2862, 1630, 1554, 1484, 1440, 1399, 1357, 1332, 1262, 1082, 1067, 1009, 852, 840, 822, 782, $743 \mathrm{~cm}^{-1}$. HRMS (ESI, $\left.m / z\right)$ : Calcd for $\mathrm{C}_{21} \mathrm{H}_{16} \mathrm{BrN}_{2}$ $\left(\mathrm{M}+\mathrm{H}^{+}\right)$375.0497, found 375.0474 .

8-(3-Chlorophenyl)-10,11-dihydro-9H-cyclopenta[a][4,7]phenanthroline (4b). Mp 194-196 ${ }^{\circ} \mathrm{C} ;{ }^{1} \mathrm{H} \mathrm{NMR}\left(\mathrm{CDCl}_{3}, 400 \mathrm{MHz}\right): \delta_{\mathrm{H}} 2.30 \sim 2.38\left(\mathrm{~m}, 2 \mathrm{H}, \mathrm{CH}_{2}\right), 3.30\left(\mathrm{t}, J 7.6 \mathrm{~Hz}, 2 \mathrm{H}, \mathrm{CH}_{2}\right), 3.78$ $\left(\mathrm{t}, J 7.2 \mathrm{~Hz}, 2 \mathrm{H}, \mathrm{CH}_{2}\right), 7.45 \sim 7.49(\mathrm{~m}, 2 \mathrm{H}, \mathrm{ArH}), 7.61(\mathrm{dd}, J 8.4 \mathrm{~Hz}, J \cdot 4.4 \mathrm{~Hz}, 1 \mathrm{H}, \mathrm{ArH})$, $7.76 \sim 7.80$ (m, 1H, ArH), 7.93 (s, 1H, ArH), 8.21 (d, J 9.2 Hz, 1H, ArH), 8.33 (d, J 9.2 Hz, 1H, ArH), 9.00 9.03 (m, 2H, ArH). IR (KBr): v 3063, 2956, 2923, 2851, 1584, 1564, 1517, 1485, 1479, 1461, 1442, 1421, 1403, 1332, 1253, 1079, 833, 802, 777, 722, $703 \mathrm{~cm}^{-1}$. HRMS (ESI, $\mathrm{m} / \mathrm{z})$ : Calcd for $\mathrm{C}_{21} \mathrm{H}_{16} \mathrm{ClN}_{2}\left(\mathrm{M}+\mathrm{H}^{+}\right) 331.1002$, found 331.0964 .

8-(4-Chlorophenyl)-10,11-dihydro-9H-cyclopenta[a][4,7]phenanthroline (4c). Mp 228-229 ${ }^{\circ} \mathrm{C}$, (Lit. ${ }^{10}$ m.p. $\left.227 \sim 228{ }^{\circ} \mathrm{C}\right) ;{ }^{1} \mathrm{H}$ NMR $\left(\mathrm{CDCl}_{3}, 400 \mathrm{MHz}\right): \delta_{\mathrm{H}} 2.31 \sim 2.38\left(\mathrm{~m}, 2 \mathrm{H}, \mathrm{CH}_{2}\right), 3.29(\mathrm{t}$, $\left.J 7.6 \mathrm{~Hz}, 2 \mathrm{H}, \mathrm{CH}_{2}\right), 3.77\left(\mathrm{t}, J 7.2 \mathrm{~Hz}, 2 \mathrm{H}, \mathrm{CH}_{2}\right), 7.51(\mathrm{~d}, J 8.4 \mathrm{~Hz}, 2 \mathrm{H}, \mathrm{ArH}), 7.60$ (dd, J 8.4 Hz, J' $4.4 \mathrm{~Hz}, 1 \mathrm{H}, \mathrm{ArH}), 7.87$ (d, J 8.4 Hz, 2H, ArH), 8.20 (d, J 9.2 Hz, 1H, ArH), 8.32 (d, J 9.2 Hz, 1H, ArH), 9.00 9.02 (m, 2H, ArH). IR (KBr): v 3033, 2954, 2943, 2930, 2862, 1584, 1556, $1515,1484,1441,1400,1363,1332,1279,1261,1179,1087,1012,854,839,822,782,745 \mathrm{~cm}^{-}$ 1. HRMS (ESI, $m / z)$ : Calcd for $\mathrm{C}_{21} \mathrm{H}_{16} \mathrm{ClN}_{2}\left(\mathrm{M}+\mathrm{H}^{+}\right) 331.1002$, found 331.1037.

8-(3-Fluorophenyl)-10,11-dihydro-9H-cyclopenta[a][4,7]phenanthroline (4d). Mp 161-162 ${ }^{\circ} \mathrm{C} ;{ }^{1} \mathrm{H} \mathrm{NMR}\left(\mathrm{CDCl}_{3}, 400 \mathrm{MHz}\right): \delta_{\mathrm{H}} 2.30 \sim 2.37\left(\mathrm{~m}, 2 \mathrm{H}, \mathrm{CH}_{2}\right), 3.31\left(\mathrm{t}, J 7.6 \mathrm{~Hz}, 2 \mathrm{H}, \mathrm{CH}_{2}\right), 3.77$ (t, J 7.2 Hz, 2H, $\mathrm{CH}_{2}$ ), 7.15 7.19 (m, 1H, ArH), 7.47 7.52 (m, 1H, ArH), 7.60 (dd, J 8.4 Hz, J' $4.4 \mathrm{~Hz}, 1 \mathrm{H}, \mathrm{ArH}), 7.62 \sim 7.70(\mathrm{~m}, 2 \mathrm{H}, \mathrm{ArH}), 8.20$ (d, J $9.2 \mathrm{~Hz}, 1 \mathrm{H}, \mathrm{ArH}), 8.33$ (d, J 9.2 Hz, 1H, ArH), 8.99 9.02 (m, 2H, ArH).

IR (KBr): v 3063, 2978, 2956, 1613, 1583, 1565, 1518, 1486, 1461, 1443, 1402, 1363, 1259 , 1218, 1139, 1112, 959, 877, 832, 788, $703 \mathrm{~cm}^{-1}$. HRMS (ESI, $\left.m / z\right)$ : Calcd for $\mathrm{C}_{21} \mathrm{H}_{16} \mathrm{FN}_{2}(\mathrm{M}+$ $\left.\mathrm{H}^{+}\right)$315.1298, found 315.1314 .

8-(4-Fluorophenyl)-10,11-dihydro-9H-cyclopenta[a][4,7]phenanthroline (4e). Mp 189 190 ${ }^{\circ} \mathrm{C}$, (Lit. ${ }^{10}$ m.p. 189 190 $\left.{ }^{\circ} \mathrm{C}\right) ;{ }^{1} \mathrm{H}$ NMR $\left(\mathrm{CDCl}_{3}, 400 \mathrm{MHz}\right): \delta_{\mathrm{H}} 2.29 \sim 2.37\left(\mathrm{~m}, 2 \mathrm{H}, \mathrm{CH}_{2}\right), 3.29(\mathrm{t}$, $\left.J 7.6 \mathrm{~Hz}, 2 \mathrm{H}, \mathrm{CH}_{2}\right), 3.76\left(\mathrm{t}, J 7.2 \mathrm{~Hz}, 2 \mathrm{H}, \mathrm{CH}_{2}\right), 7.21(\mathrm{t}, J 8.8 \mathrm{~Hz}, 2 \mathrm{H}, \mathrm{ArH}), 7.59$ (dd, J 8.4 Hz, J’ 4.4 Hz, 1H, ArH), 7.89 7.91 (m, 2H, ArH), 8.19 (d, J 9.2 Hz, 1H, ArH), 8.32 (d, J 9.2 Hz, 1H, ArH), 8.99 9.01 (m, 2H, ArH). IR (KBr): v 3044, 2955, 2943, 2861, 1598, 1559, 1506, 1485 , $1443,1403,1332,1227,1211,1168,1106,860,837,810,781,747 \mathrm{~cm}^{-1}$.

10,11-Dihydro-8-p-tolyl-9H-cyclopenta[a][4,7]phenanthroline (4f). Mp 125 126 ${ }^{\circ} \mathrm{C} ;{ }^{1} \mathrm{H}$ $\mathrm{NMR}\left(\mathrm{CDCl}_{3}, 400 \mathrm{MHz}\right): \delta_{\mathrm{H}} 2.28 \sim 2.35\left(\mathrm{~m}, 2 \mathrm{H}, \mathrm{CH}_{2}\right), 2.45\left(\mathrm{~s}, 3 \mathrm{H}, \mathrm{CH}_{3}\right), 3.30(\mathrm{t}, J 7.6 \mathrm{~Hz}, 2 \mathrm{H}$, $\mathrm{CH}_{2}$ ), 3.75 (t, J 7.2 Hz, 2H, $\mathrm{CH}_{2}$ ), 7.34 (t, J 8.0 Hz, 2H, ArH), 7.58 (dd, J 8.4 Hz, J' 4.4 Hz, 1H, ArH), $7.81(\mathrm{~d}, J 8.0 \mathrm{~Hz}, 2 \mathrm{H}, \mathrm{ArH}), 8.18(\mathrm{~d}, J 9.2 \mathrm{~Hz}, 1 \mathrm{H}, \mathrm{ArH}), 8.32$ (d, J 9.2 Hz, 1H, ArH), 8.96 8.99 (m, 2H, ArH). IR (KBr): v 3042, 2948, 2920, 2851, 1609, 1586, 1555, 1485, 1444, 1401, 1363, 1331, 1279, 1262, 1181, 1117, 1023, 835, 821, 779, $742 \mathrm{~cm}^{-1}$. HRMS (ESI, $\left.m / z\right)$ : Calcd for $\mathrm{C}_{22} \mathrm{H}_{19} \mathrm{~N}_{2}\left(\mathrm{M}+\mathrm{H}^{+}\right)$311.1548, found 311.1567.

10,11-Dihydro-8-(4-methoxyphenyl)-9H-cyclopenta[a][4,7]phenanthroline (4g). Mp 179-180 ${ }^{\circ} \mathrm{C} ;{ }^{1} \mathrm{H} \mathrm{NMR}\left(\mathrm{CDCl}_{3}, 400 \mathrm{MHz}\right): \delta_{\mathrm{H}} 2.27 \sim 2.35\left(\mathrm{~m}, 2 \mathrm{H}, \mathrm{CH}_{2}\right), 3.30\left(\mathrm{t}, J 7.6 \mathrm{~Hz}, 2 \mathrm{H}, \mathrm{CH}_{2}\right), 3.24$ 
(t, J 7.2 Hz, 2H, $\left.\mathrm{CH}_{2}\right), 3.90\left(\mathrm{~s}, 3 \mathrm{H}, \mathrm{CH}_{3} \mathrm{O}\right), 7.04 \sim 7.08(\mathrm{~m}, 2 \mathrm{H}, \mathrm{ArH}), 7.57$ (dd, J 8.4 Hz, J' 4.4 $\mathrm{Hz}, 1 \mathrm{H}, \mathrm{ArH}), 7.88 \sim 7.90$ (m, 2H, ArH), 8.18 (d, J $9.2 \mathrm{~Hz}, 1 \mathrm{H}, \mathrm{ArH}), 8.32$ (d, J 9.2 Hz, 1H, ArH), 8.96 8.99 (m, 2H, ArH). IR (KBr): $v$ 2950, 2909, 2836, 1603, 1556, 1504, 1481, 1442 , 1400, 1362, 1282, 1250, 1173, 1026, 841, 824, 784, 760, $749 \mathrm{~cm}^{-1}$. HRMS (ESI, $\left.\mathrm{m} / \mathrm{z}\right)$ : Calcd for $\mathrm{C}_{22} \mathrm{H}_{19} \mathrm{~N}_{2} \mathrm{O}\left(\mathrm{M}+\mathrm{H}^{+}\right) 327.1517$, found 327.1514 .

10,11-Dihydro-8-(2,3-dimethoxyphenyl)-9H-cyclopenta[a][4,7]phenanthroline $\quad(4 \mathrm{~h}) . \quad \mathrm{Mp}$ 206-207 ${ }^{\circ} \mathrm{C} ;{ }^{1} \mathrm{H}$ NMR $\left(\mathrm{CDCl}_{3}, 400 \mathrm{MHz}\right): \delta_{\mathrm{H}} 2.26 \sim 2.31\left(\mathrm{~m}, 2 \mathrm{H}, \mathrm{CH}_{2}\right), 3.08(\mathrm{t}, J 7.6 \mathrm{~Hz}, 2 \mathrm{H}$, $\left.\mathrm{CH}_{2}\right), 3.59$ (s, 3H, $\left.\mathrm{CH}_{3} \mathrm{O}\right), 3.80\left(\mathrm{t}, J 7.2 \mathrm{~Hz}, 2 \mathrm{H}, \mathrm{CH}_{2}\right), 3.95\left(\mathrm{~s}, 3 \mathrm{H}, \mathrm{CH}_{3} \mathrm{O}\right), 7.04(\mathrm{~d}, J 8.0 \mathrm{~Hz}, 1 \mathrm{H}$, ArH), 7.11 (d, J 7.6 Hz, 1H , ArH), 7.20 7.23 (m, 1H, ArH), 7.61 (dd, J 8.8 Hz, J' 4.4 Hz, 1H, ArH), 8.19 (d, J 9.2 Hz, 1H, ArH), 8.35 (d, J 9.2 Hz, 1H, ArH), 9.00 (d, J 3,2 Hz, 1H, ArH), 9.06 (d, J $8.4 \mathrm{~Hz}, 1 \mathrm{H}, \mathrm{ArH}) . \mathrm{IR}(\mathrm{KBr}): v 2965,2930,2835,1582,1561,1516,1487,1470,1441$, 1405, 1381, 1365, 1266, 1227, 1064, 1040, 990, 857, 798, $756 \mathrm{~cm}^{-1}$. HRMS (ESI, $\left.\mathrm{m} / z\right)$ ): Calcd for $\mathrm{C}_{23} \mathrm{H}_{21} \mathrm{~N}_{2} \mathrm{O}_{2}\left(\mathrm{M}+\mathrm{H}^{+}\right) 357.1603$, found 357.1622.

10,11-Dihydro-8-(3,4-dimethylphenyl)-9H-cyclopenta[a][4,7]phenanthroline (4i). Mp 183$184{ }^{\circ} \mathrm{C} ;{ }^{1} \mathrm{H}$ NMR $\left(\mathrm{CDCl}_{3}, 400 \mathrm{MHz}\right): \delta_{\mathrm{H}} 2.28 \sim 2.34\left(\mathrm{~m}, 2 \mathrm{H}, \mathrm{CH}_{2}\right), 2.36\left(\mathrm{~s}, 3 \mathrm{H}, \mathrm{CH}_{3}\right), 2.39(\mathrm{~s}, 3 \mathrm{H}$, $\left.\mathrm{CH}_{3}\right), 3.30\left(\mathrm{t}, J 7.6 \mathrm{~Hz}, 2 \mathrm{H}, \mathrm{CH}_{2}\right), 3.74\left(\mathrm{t}, J 7.2 \mathrm{~Hz}, 2 \mathrm{H}, \mathrm{CH}_{2}\right), 7.28(\mathrm{~d}, J 8.0 \mathrm{~Hz}, 1 \mathrm{H}, \operatorname{ArH})$, 7.55 7.61 (m, 2H, ArH), 7.72 (s, 1H, ArH), 8.18 (d, J 9.2 Hz, 1H, ArH), 8.34 (d, J 9.2 Hz, 1H, ArH), 8.97 9.00 (m, 2H, ArH). IR (KBr): v 2963, 2938, 2918, 2880, 2850, 1581, 1556, 1482, 1453, 1441, 1415, 1390, 1364, 1333, 1260, 1125, 1084, 1022, 998, 842, 827, $784 \mathrm{~cm}^{-1}$. HRMS (ESI, $m / z)$ : Calcd for $\mathrm{C}_{23} \mathrm{H}_{21} \mathrm{~N}_{2}\left(\mathrm{M}+\mathrm{H}^{+}\right)$325.1705, found 325.1721 .

8-(3,4-Methylenedioxyphenyl)-10,11-dihydro-9H-cyclopenta[a][4,7]phenanthroline $(\mathbf{4 j}) . \mathrm{Mp}$ 182-183 ${ }^{\circ} \mathrm{C}$, (Lit. ${ }^{10}$ m.p. $\left.183 \sim 184{ }^{\circ} \mathrm{C}\right) ;{ }^{1} \mathrm{H}$ NMR $\left(\mathrm{CDCl}_{3}, 400 \mathrm{MHz}\right): \delta_{\mathrm{H}} 2.28 \sim 2.35\left(\mathrm{~m}, 2 \mathrm{H}, \mathrm{CH}_{2}\right)$, $3.29\left(\mathrm{t}, J 7.6 \mathrm{~Hz}, 2 \mathrm{H}, \mathrm{CH}_{2}\right), 3.75\left(\mathrm{t}, J 7.2 \mathrm{~Hz}, 2 \mathrm{H}, \mathrm{CH}_{2}\right), 6.05\left(\mathrm{~s}, 2 \mathrm{H}, \mathrm{CH}_{2}\right), 6.97(\mathrm{~d}, J 8.0 \mathrm{~Hz}, 1 \mathrm{H}$, ArH), 7.41 (dd, J 8.0 Hz, J' 2.0 Hz, 1H, ArH), 7.46 (d, J 1.2 Hz, 1H, ArH), 7.58 (dd, J 8.0 Hz, J' $4.4 \mathrm{~Hz}, 1 \mathrm{H}, \mathrm{ArH}), 8.18$ (d, J 9.2 Hz, 1H, ArH), 8.31 (d, J 9.2 Hz, 1H, ArH), 8.97 9.00 (m, 2H, ArH). IR (KBr): $v$ 2972, 2953, 2905, 2884, 1585, 1557, 1501, 1482, 1441, 1404, 1342, 1252 , $1238,1101,1037,930,838,821,786,746 \mathrm{~cm}^{-1}$. HRMS (ESI, $\left.\mathrm{m} / z\right)$ : Calcd for $\mathrm{C}_{22} \mathrm{H}_{17} \mathrm{~N}_{2} \mathrm{O}_{2}(\mathrm{M}+$ $\left.\mathrm{H}^{+}\right) 341.1290$, found 341.1288 .

\section{Acknowledgements}

We are grateful to the National Natural Science foundation of China (20802061), A Project Funded by the Priority Academic Program Development of Jiangsu Higher Education Institutions and Qing Lan Project (08QLT001, 10QLD008) of Jiangsu Education Committee for financial support. 


\section{References}

1. (a) Vijjay, N. C.; Rajesh, A. U.; Vinod, S.; Bindu, A. R.; Sreekanth, J. S.; Lakshmi, B. Acc. Chem. Res. 2003, 36, 899. (b) Chen, Y. K.; Walsh, P. J. J. Am. Chem. Soc. 2004, 126, 3702. (c) Tietze, L. F. Chem. Rev. 1996, 96, 115.

2. (a) Liéby-Muller, F.; Constantieux, T.; Jean Rodriguez, J. J. Am. Chem. Soc. 2005, 127, 17176. (b) Bandgar, B. P.; More, P. E.; Kamble, V. T.; Totre, J. V. Arkivoc 2008, (xv), 1. (c) Chebanov, V. A.; Saraev, V. E.; Desenko, S. M.; Chernenko, V. N.; Knyazeva, I. V.; Groth, U.; Glasnov, T. N.; Kappe, C. O. J. Org. Chem. 2008, 73, 5110. (d) Bantval, S. H; Kani, V. M.; Balladka, K. S.; Boja, P. Synth. Commun. 2005, 35, 333. (e) Wang, X. S.; Li, Q.; Yao, C. S.; Tu, S. J. Eur. J. Org. Chem. 2008, (20), 3513. (f) Srinivasu V. N. V.; Rajashekar, B. L.; Srinivas, K. J. Heterocycl. Chem. 2010, 47, 687.

3. (a) Biology; Dolle, R. E.; Bourdonnec, B. L.; Goodman, A. J.; Morales, G. A.; Thomas, C. J.; Zhang, W. J. Comb. Chem. 2008, 10, 753. (b) Hassan, S.; Maryam, B. Synth. Commun. 2010, 40, 257. (c) Sapi, J.; Laronze, J. Y. Arkivoc 2004, (vii), 208.

4. Devereux, M.; O'Shea, D.; Kellett, A.; McCann, M.; Walsh, M.; Egan, D.; Deegan, C.; Kedziora, K.; Rosair, G.; Mueller-Bunz, H. J. Inorg. Biochem. 2007, 101, 881.

5. Sharma, K. V.; Sharma, V.; Dubey, R. K.; Tripathi, U. N. J. Coord. Chem. 2009, 62, 493.

6. Margiotta, N.; Papadia, P.; Lazzaro, F.; Crucianelli, M.; De Angelis, F.; Pisano, C.; Vesci, L.; Natile, G. J. Med. Chem. 2005, 48, 7821.

7. 7 Gameiro, P.; Rodrigues, C.; Baptista, T.; Sousa, I.; de Castro, B. Int. J. Pharm. 2007, 334, 129.

8. He, Q. Z.; Yang, J.; Min, H.; Li, H. X. Mater. Lett. 2006, 60, 317.

9. Wesselinova, D.; Neykov, M.; Kaloyanov, N.; Toshkova, R.; Dimitrov, G. Eur. J. Med. Chem. 2009, 44, 2720.

10. Tereshko, A. B.; Kozlov, N. G.; Gusak, K. N. Russ. J. Gen. Chem. 2003, 73, 1619.

11. (a) Wang, X. S.; Li, Q.; Wu, J. R.; Li, Y. L.; Yao, C. S.; Tu, S. J. Synthesis 2008, 1902. (b) Wang, X. S.; Li, Q.; Wu, J. R.; Tu, S. J. J. Comb. Chem. 2009, 11, 433. (c) Wang, X. S.; Zhou, J.; Yin, M. Y.; Yang, K,; Tu, S. J. J. Comb. Chem. 2010, 12, 266. (d) Wang, X. S.; Li, Q.; Wu, J. R.; Li, Y. L. Synth. Commun. 2009, 39, 702.

12. Wang, X. S.; Zhou, J.; Yin, M. Y.; Yang, K,; Tu, S. J. J. Heterocycl. Chem. 2010, 47, 873. 